\title{
Different Structural Conformers of Monomeric $\alpha$-Synuclein Identified after Lyophilizing and Freezing
}

\author{
Amberley D. Stephens, ${ }^{\dagger}$ No Nezhda Nespovitaya, ${ }^{\dagger}$ Maria Zacharopoulou, ${ }^{\dagger}$ Clemens F. Kaminski, ${ }^{\dagger}$ \\ Jonathan J. Phillips, ${ }^{\ddagger}$ and Gabriele S. Kaminski Schierle* ${ }^{* \dagger}$ \\ ${ }^{\dagger}$ Departmtent of Chemical Engineering and Biotechnology, University of Cambridge, Philippa Fawcett Drive, Cambridge, CB3 0AS, \\ U.K. \\ ${ }^{\ddagger}$ Living Systems Institute, University of Exeter, Stocker Road, Exeter, EX4 4QD, U.K.
}

\section{Supporting Information}

ABSTRACT: Understanding the mechanisms behind amyloid protein aggregation in diseases, such as Parkinson's and Alzheimer's disease, is often hampered by the reproducibility of in vitro assays. Yet, understanding the basic mechanisms of protein misfolding is essential for the development of novel therapeutic strategies. We show here, that for the amyloid protein $\alpha$-synuclein (aSyn), a protein involved in Parkinson's disease (PD), chromatographic buffers and storage conditions can significantly interfere with the overall structure of the protein and thus affect protein aggregation kinetics. We apply several biophysical and biochemical methods, including size exclusion chromatography (SEC), dynamic light scattering (DLS), and atomic force microscopy (AFM), to characterize the high molecular weight conformers formed during protein purification and storage. We further apply hydrogen/deuterium-exchange mass spectrometry (HDX-MS) to characterize the monomeric form of aSyn and reveal a thus far unknown structural component of aSyn at the C-terminus of the protein. Furthermore, lyophilizing the protein greatly affected the overall structure of this monomeric conformer. We conclude from this study that structural polymorphism may occur under different storage conditions, but knowing the structure of the majority of the protein at the start of each experiment, as well as the factors that may influence it, may pave the way to an improved understanding of the mechanism leading to aSyn pathology in PD.
$\mathrm{T}$ he study of protein misfolding and amyloid fibril formation is important in the field of neurodegeneration, including Parkinson's, Alzheimer's, Huntington's, and prion diseases, as well as other misfolding diseases, such as antibody light chain amyloidosis and diabetes, where incorrectly folded proteins also form insoluble $\beta$-sheets. ${ }^{1-3}$ Understanding amyloid fibril formation is similarly important in the field of nanomedicine, for instance, in the synthesis of structural tissue scaffolds, whereby artificial self-assembling amyloids can be utilized for their tensile strength and ease of functionalization. ${ }^{4}$ Unfortunately, tracking the transformation of initially soluble monomeric species into insoluble amyloid fibrils is difficult because experimental conditions and sample heterogeneity greatly affect the reproducibility of aggregation assays. Several fluorescence-based methods are in use to track the formation of amyloid fibrils, such as thioflavin T (ThT), Congo Red, and $\mathrm{N}$ arylaminonaphthalenesulfonate (ANS)-based assays ${ }^{5}$ and, more recently, the intrinsic fluorescence-based assay. ${ }^{6,7}$ Much work has been undertaken to increase the reproducibility of experiments investigating amyloid fibril formation kinetics. The addition of salts, metals, alteration of $\mathrm{pH}$ or temperature greatly affects the aggregation rate of amyloid proteins and the addition of sodium dodecyl sulfate (SDS) or glass beads has been shown to reduce-well-to-well variability of aggregation kinetic curves from ThT-based assays. ${ }^{8-10}$ It is thus of foremost importance to have a clear understanding of the impact of purification protocols, including the choice of buffers, protein purity, and protein storage format.

We have studied the amyloid protein, $\alpha$-synuclein (aSyn), which is involved in Parkinson's disease (PD). Monomeric aSyn is an intrinsically disordered (IDP) soluble protein formed of an N-terminus lipid binding domain (aa 1-60), a hydrophobic, so-called nonamyloid-component (NAC) region (aa 61-95), which increases the aggregation propensity of the protein, and a negatively charged $\mathrm{C}$-terminus domain that modulates aggregation (aa 96-140). ${ }^{11}$ Like many other amyloid proteins, so far, the mechanistic pathway from soluble unstructured aSyn to insoluble fibrils has not been fully elucidated. To achieve this goal, one requires reliable and reproducible aggregation assays and accordingly an understanding of the conformational states of the protein at the start of the assay. However, at present, there are many protocols

Received: March 20, 2018

Accepted: May 11, 2018

Published: May 11, 2018 
discussed in the literature (see Table 1) on how to purify and store recombinant aSyn. The effect of protein storage on

Table 1. Overview over Different Protocols Currently Used for Purification of Recombinant aSyn Protein

\begin{tabular}{|c|c|c|c|}
\hline protein isolation & $\begin{array}{l}\text { chromatography } \\
\text { steps }\end{array}$ & storage & ref \\
\hline homogenized and boiled & IEX, RP-HPLC & lyophilized & 15 \\
\hline $\begin{array}{l}\text { cell lysate ammonium } \\
\text { sulfate precipitation }\end{array}$ & SEC, IEX & not stated & 16 \\
\hline $\begin{array}{l}\text { cell lysate ammonium } \\
\text { sulfate precipitation }\end{array}$ & IEX, SEC & lyophilized & 17 \\
\hline cell lysate acid precipitation & IEX & not stated & 18 \\
\hline $\begin{array}{l}\text { periplasmic lysis by osmotic } \\
\text { shock }\end{array}$ & IEX & $\begin{array}{l}\text { lyophilized, SEC } \\
\text { before use }\end{array}$ & 12 \\
\hline $\begin{array}{l}\text { freeze/thaw, sonicating and } \\
\text { boiling }\end{array}$ & $\begin{array}{l}\text { IEX, SEC, IEX, } \\
\text { SEC }\end{array}$ & $\begin{array}{l}\text { frozen, SEC before } \\
\text { use }\end{array}$ & 19 \\
\hline $\begin{array}{l}\text { periplasmic lysis by osmotic } \\
\text { shock }\end{array}$ & IEX, HIC & $\begin{array}{l}\text { lyophilized, dialyzed } \\
\text { before use }\end{array}$ & 13 \\
\hline
\end{tabular}

protein aggregation kinetics has so far not been investigated. In the current study, we used the protocol described by Huang et al. and Campioni et al., applying periplasmic lysis, ion exchange chromatography (IEX), and hydrophobic interaction chromatography (HIC), to purify recombinant aSyn. ${ }^{12,13}$ Importantly, this protocol does not include a heating step, which may lead to partial protein degradation that may affect assay variability. ${ }^{14}$

After purification, we dialyzed the protein into different buffers and then either lyophilized or froze it for storage. Lyophilization is commonly used in protein research ${ }^{20}$ since it provides for facile storage of the protein, easy reconstitution in any desired buffer solution, and does not require any timeconsuming buffer exchanges. Lyophilization involves three steps: (i) freezing, (ii) primary dehydration to remove ice (sublimation), and (iii) secondary dehydration to remove traces of water (desorption). ${ }^{21}$ However, each step of the process may introduce protein damage by degradation or structural alteration or protein aggregation. ${ }^{22-27}$ Moreover, the structural recovery of proteins after lyophilization is dependent on the protein and the buffer composition, while many proteins will refold upon reconstitution in aqueous solution, some will remain misfolded or aggregated. ${ }^{28}$

Here, we investigate the impact of buffer solutions and protein storage conditions on the aggregation kinetics of recombinant human wild type (WT) aSyn by lyophilizing or freezing the protein in $\mathrm{H}_{2} \mathrm{O}$ or $20 \mathrm{mM}$ sodium phosphate $(\mathrm{NaP})$ buffer, $\mathrm{pH}$ 7.2. We show that the aggregation kinetics of aSyn are affected by lyophilization as demonstrated in a ThTbased aggregation assay. On the one hand, lyophilization of aSyn accelerates amyloid nucleation reactions, while on the other hand the elongation reaction is slowed down compared to freezing. Moreover, the plate-to-plate variance in the amount of amyloid aggregates formed is increased in the lyophilized aSyn samples when compared to the frozen samples. Although the samples were primarily monomeric, the presence of a small percentage of high molecular weight (HMW) conformers was confirmed by analytical size exclusion chromatography (SEC), dynamic light scattering (DLS), and atomic force microscopy (AFM), all of which displayed a variety of sizes and shapes of aSyn HMW conformers formed under both lyophilizing and freezing processes. We call these "HMW conformers" because currently we cannot conclude whether these are similar to what is referred to as "oligomers" in the literature. Finally, we used hydrogen-deuterium exchange mass spectrometry (HDX-MS) to probe the molecular conformation of the primarily monomeric aSyn to determine whether storage conditions affected the sub molecular structure of aSyn. Interestingly, we show that in both lyophilized and frozen aSyn samples the NAC region of aSyn was the least solvent-protected region, while residues $115-125$ and $134-140$ of the C-terminus were the most protected. We also observed that lyophilizing affects the structure of the monomeric protein, as the aSyn sample was overall less accessible to the solvent throughout the protein sequence compared to the frozen sample. We highlight the importance of a full characterization of the amyloid protein sample prior to analyzing its structure-function relationship.

\section{METHODS AND MATERIALS}

Escherichia coli Expression of aSyn and Periplasmic Lysis. The plasmid pT7-7 containing human aSyn cDNA was transformed into Escherichia coli One Shot BL21 Star (DE3) (Thermo Fisher Scientific, USA). Two liter cultures of E. coli in Lysogeny Broth (LB) containing carbenicillin $(100 \mu \mathrm{g} / \mathrm{mL})$ were grown at $37^{\circ} \mathrm{C}$ and induced for expression of aSyn with 1 $\mathrm{mM}$ isopropyl- $\beta$-thiogalactopyranoside (IPTG). The cells were pelleted by centrifugation at $7000 \mathrm{~g}$. To release the expressed aSyn, lysis of the periplasm was performed following the protocol described in ref 12 The final supernatant containing aSyn and periplasmic proteins was collected and dialyzed overnight in ion exchange (IEX) buffer A (20 mM Tris- $\mathrm{HCl}$, $\mathrm{pH} 8$ ) in SnakeSkin dialysis tubing, MWCO $10 \mathrm{kDa}$ (Thermo Fisher Scientific, USA).

aSyn Purification Using IEX and HIC. For purification of aSyn, we followed the protocol described in ref 13 . The protein was loaded onto a HiPrep QFF 16/10 anion exchange column (GE Healthcare, Sweden) and eluted against a linear gradient of IEX buffer B (20 mM Tris-HCl, $0.5 \mathrm{M} \mathrm{NaCl}, \mathrm{pH} 8)$. Fractions containing aSyn were pooled and dialyzed overnight using hydrophobic interaction chromatography (HIC) buffer A (1 M $\left(\mathrm{NH}_{4}\right)_{2} \mathrm{SO}_{4}, 50 \mathrm{mM}$ Bis-Tris, $\mathrm{pH} \mathrm{7)}$ in Slide-A-Lyzer dialysis cassettes (10 kDa MWCO; Thermo Fischer Scientific, USA). The dialyzed protein was loaded onto a HiPrep Phenyl FF 16/ 10 (High Sub) hydrophobic interaction column (GE Healthcare, Sweden) and eluted using HIC buffer B (50 mM Bis-Tris, $\mathrm{pH}$ 7). IEX and HIC were performed on an ÄKTA Pure (GE Healthcare, Sweden). Pooled IEX and HIC fractions were analyzed on a $4-12 \%$ Bis-Tris gel by SDS-PAGE and stained with Coomassie blue. Quantitative analysis of protein purity was performed in FIJI image analysis software ${ }^{29}$ by profiling protein band intensity of the stained gel. aSyn was purified twice for these experiments and reversed phase-high pressure liquid chromatography (RP-HPLC) determined aSyn to be $85.22 \%$ pure in purification batch 1 and $90.71 \%$ pure in purification batch 2. Amino acid analysis confirmed the composition of the purified WT human aSyn (Table S1).

aSyn Storage Using Either Lyophilization or Freezing at $-80^{\circ} \mathrm{C}$. The fractions containing aSyn eluted from the HIC column were pooled together and dialyzed extensively using either $\mathrm{H}_{2} \mathrm{O}$ or $20 \mathrm{mM} \mathrm{Na} \mathrm{HPO}_{4} \mathrm{pH} 7.2(\mathrm{NaP})$ in Slide-ALyzer dialysis cassettes (10 kDa MWCO). The dialyzates were split into $1 \mathrm{~mL}$ aliquots. One aliquot of aSyn in $\mathrm{NaP}$ buffer was directly placed at $-80{ }^{\circ} \mathrm{C}$, and one aliquot of either aSyn in $\mathrm{NaP}$ buffer or $\mathrm{H}_{2} \mathrm{O}$ was frozen in liquid nitrogen, lyophilized in a LyoQuest 85 freeze-dryer (Telstar, Spain), and stored at -80 ${ }^{\circ} \mathrm{C}$.

Analytical Size Exclusion Chromatography (SEC). SEC analysis was performed on an Agilent 1260 Infinity HPLC 
system (Agilent Technologies LDA UK Limited, UK) equipped with an autosampler and a diode-array detector using a BioSepSEC-2000s column (Phenomenex, UK) in phosphate-buffered saline (Gibco PBS, Thermo Fischer Scientific UK LTD, UK) at $1 \mathrm{~mL} / \mathrm{min}$ flow-rate. The elution profile was monitored by UV absorption at 220 and $280 \mathrm{~nm}$. For the SEC-HPLC analysis, the directly frozen aSyn samples were thawed at room temperature, samples lyophilized in $\mathrm{H}_{2} \mathrm{O}$ were resuspended in $20 \mathrm{mM} \mathrm{NaP}$ $\mathrm{pH} 7.2$, and aSyn lyophilized in $20 \mathrm{mM} \mathrm{NaP} \mathrm{pH} \mathrm{7.2,} \mathrm{was}$ resuspended in $\mathrm{H}_{2} \mathrm{O}$. All aSyn samples were diluted to $100 \mu \mathrm{M}$ and filtered through a $0.22 \mu \mathrm{m}$ filter (Millipore (U.K.) Limited, UK). The SEC injection volume was $50 \mu \mathrm{L}$, six injections were made for both frozen and lyophilized samples from purification batches 1 and 2. To estimate the molecular weight of aSyn species by their column retention times, a standard protein mixture (PN 69385, Sigma-Aldrich) was used to calibrate the BioSep-SEC-2000s column.

Dynamic Light Scattering (DLS). Samples were analyzed on a Zetasizer Nano S (Malvern Instruments Ltd., UK). The DLS instrument was equipped with a Peltier temperature controller set at $+25{ }^{\circ} \mathrm{C}$ temperature. Disposable microcuvettes (Malvern Instruments Ltd., UK) were used for size measurements which were conducted at a scattering angle of $173^{\circ}$. Every sample was measured at least seven times with two or three repeats for samples from purification batches 1 and 2 .

Atomic Force Microscopy (AFM). Ten microliters of 100 $\mu \mathrm{M}$ solutions of frozen or lyophilized aSyn were incubated on a freshly cleaved mica surface for $30 \mathrm{~min}$. The mica was washed three times in deionized $\mathrm{H}_{2} \mathrm{O}$ to remove lose protein and dried under a stream of nitrogen. Images covering a field of view of 1 $\mu \mathrm{m} \times 1 \mu \mathrm{m}$ were acquired in "Peak Force Quantitative Nanomechanical Property mode” using ScanAsyst Air probes (BioScope Resolve, Bruker AXS GmbH). Images were acquired at a scan rate of $1 \mathrm{~Hz}$ using a peak force $\leq 1.5 \mathrm{nN}$. The peak force amplitude was set to $70 \mathrm{~nm}$. Images were flattened using the NanoScope Analysis software, version 1.8, before being exported for further analysis. Images were analyzed using the ICY imaging software (http://icy.bioimageanalysis.org/) and were gray rendered before analysis. The area of oligomers was then calculated using the "Connected Components" plugin. The gray scale 8-bit images were given a threshold of 50/256 to remove background and the minimum size detection was 1 pixel to allow detection of very small conformers with height profiles, 1 pixel $=1.95 \mathrm{~nm}$. A group of connected pixels was detected as a cluster (one aggregate). Its area was attributed to the area of the respective HMW conformer.

Hydrogen/Deuterium-Exchange Mass Spectrometry (HDX-MS). Hydrogen-deuterium exchange was performed using an HDX Manager (Waters, US) equipped with a CTC PAL sample handling robot (LEAP Technologies, US). Samples of WT aSyn in protonated aqueous buffer $(20 \mathrm{mM}$ $\mathrm{NaP}, \mathrm{pH} 4.00)$ were diluted 20 -fold into deuterated buffer $(20$ $\mathrm{mM} \mathrm{NaP}, \mathrm{pD} 4.00)$ at $20{ }^{\circ} \mathrm{C}$, and thus hydrogen/deuteriumexchange was initiated. The buffer $\mathrm{pH}$ was adjusted to $\mathrm{pH} 4.00$ using $1 \mathrm{M} \mathrm{HCl}$ and monitored with a $\mathrm{pH}$ meter. The protein was incubated for various time points, ranging from $30 \mathrm{~s}$ to 50 min. Hydrogen exchange was arrested by mixing the protein solution $1: 1$ with a prechilled quench buffer $(20 \mathrm{mM} \mathrm{NaP}, 8 \mathrm{M}$ Urea, $\mathrm{pH} 2.45$ at $20^{\circ} \mathrm{C}$ ). The protein was then digested into peptides on an Enzymate immobilized pepsin column (Waters, US) and the peptides were trapped and then separated on C18 columns (VanGuard $2.1 \times 5 \mathrm{~mm}$ ACQUITY BEH and $1 \times 100$ mm ACQUITY BEH $1.7 \mu \mathrm{m}$, respectively, Waters, US) with a linear gradient of acetonitrile (3-40\%) supplemented with $0.1 \%$ formic acid. Peptides were analyzed with a Synapt G2-si mass spectrometer (Waters, US). Peptide mapping of aSyn, where peptides were identified by MS fragmentation, was performed prior to the hydrogen exchange experiments and analyzed using ProteinLynx Global Server- PLGS (Waters, US). Peptide mapping of aSyn yielded coverage of $100 \%$ of aSyn with a high degree of redundancy (Figure S6). Peptides were identified by $\mathrm{MS}^{\mathrm{E}}$ fragmentation with ProteinLynx Global Server (Waters, US). Deuterium incorporation was measured in DynamX 3.0 (Waters, US) and the data pertaining to deuterium uptake were analyzed and visualized in MatLab (Mathworks, UK) and Excel (Microsoft, US). No correction was made for back-exchange. The mean deuteration level per amino acid (represented on Figure S8) was calculated according to

$$
\bar{M}_{j}=\frac{1}{n} \sum_{1}^{n} \frac{1}{q} \sum_{0}^{t}\left(m_{i}^{t}-m_{i}^{0}\right)
$$

where $\bar{M}_{j}$ is the mean deuteration level at amino acid residue $j$, $n$ is the number of overlapping peptides, $q$ is the number of exchangeable amides for peptide $i, m_{i}^{t}$ is the isotopic weighted midpoint at labeling time $t$, and $m_{i}^{0}$ is the midpoint at time 0 (no deuteration).

Thioflavin-T (ThT) Binding Assay in 96-Well Plates. Ten micromolar ThT was added to $150 \mu \mathrm{L}$ of $100 \mu \mathrm{M}$ aSyn in $20 \mathrm{mM} \mathrm{Na} \mathrm{HPO}_{4} \mathrm{pH} 7.2$ from lyophilized and frozen aSyn samples. All samples were filtered through $0.22 \mu \mathrm{m}$ filters before loading onto nonbinding, clear bottom, black 96-well plates (PN 655906 Greiner Bio-One GmbH, Germany). The plates were sealed with an Ampliseal transparent microplate sealer (Grenier Bio-One $\mathrm{GmbH}$, Germany). Fluorescence measurements were taken with an Envision 2104 Multilabel Reader (PerkinElmer, Finland). The temperature was set to 37 ${ }^{\circ} \mathrm{C}$ at the bottom and $39{ }^{\circ} \mathrm{C}$ at the top of the plate to prevent condensation. The plates were incubated with orbital shaking at $300 \mathrm{rpm}$ between the readings. The readings of ThT fluorescence intensity at $486 \mathrm{~nm}$ were collected every $16 \mathrm{~min}$ in the top excitation/emission mode and at a focal height of 5.5 $\mathrm{mm}$. Excitation was at $440 \mathrm{~nm}$ with 2 flashes using $10 \%$ of the excitation light. ThT assays were repeated 3 times $(2 \times$ using purification batch 1 and $1 \times$ using purification batch 2 ) using six wells for each condition. Data were normalized to the well with the maximum fluorescence intensity for each plate. The kinetic curves of the averaged raw data from the ThT fluorescence were fitted to a sigmoidal curve and the nucleation rate $k_{1}$ and the elongation rate $k_{2}$ were calculated from the Finke-Watzky two-step model (eq 2). ${ }^{8,30}[A]_{0}$ refers to the ThT fluorescence (a.u.) at time 0 , and $[B]_{\mathrm{t}}$ refers to the ThT fluorescence (a.u.) of the fibrils formed at time $t$

$$
[B]_{t}=[A]_{0}-\frac{\frac{k_{1}}{k_{2}}+[A]_{0}}{1+\frac{k_{1}}{k_{2}[A]_{0}} \exp \left(k_{1}+k_{2}[A]_{0} t\right)}
$$

\section{RESULTS}

Lyophilization Influences aSyn Aggregation Kinetics. The kinetics of aSyn aggregation are affected by many environmental factors, including buffer composition, temperature, $\mathrm{pH}$, the presence of an air-water interface, hydrophobic surfaces or metals, as well as the presence of preformed 
oligomers. ${ }^{13,31-35}$ We first examined whether lyophilizing or freezing aSyn had an influence on the variability of aggregation kinetics measured by ThT since ThT-based assays are frequently used to monitor amyloid fibril formation. ${ }^{8,36-38}$

We optimized the purification process of human WT aSyn, starting with the protocol by Huang et al., using periplasmic lysis and IEX. ${ }^{12}$ aSyn was $78.3 \%$ pure after IEX as shown by sodium dodecyl sulfate polyacrylamide gel electrophoresis (SDS-PAGE) (Figure S1A). We next applied HIC, following the protocol by Campioni et al., which improved protein purity to $94 \%$, as assessed by SDS-PAGE (Figure S1A). ${ }^{13}$ We further used analytical RP-HPLC, which is more sensitive than SDSPAGE, to determine the purity of aSyn. After two chromatographic steps (IEX and HIC), aSyn reached a purity of $85.2 \%$ in the purification batch 1 and $90.7 \%$ in the purification batch 2 , as quantified by peak integration from RP-HPLC traces (Figure S1B). The identity of human WT aSyn was confirmed by amino acid analysis (Table S1). We dialyzed the protein in either a commonly used fibrillization buffer, $20 \mathrm{mM} \mathrm{NaP} \mathrm{pH}$ 7.2, or in $\mathrm{H}_{2} \mathrm{O}$. After this, we either lyophilized or froze the protein at $-80{ }^{\circ} \mathrm{C}$ until further use.

Before ThT-based aggregation experiments were performed, the aSyn lyophilized in NaP buffer was resuspended in $\mathrm{H}_{2} \mathrm{O}$ and both lyophilized and frozen samples were filtered through a $0.22 \mu \mathrm{m}$ membrane. The fluorescence data from the ThT-based assay exhibited sigmoidal behavior from which kinetic information could be extracted and individual well traces are shown in Figure 1A. We observed that lyophilization of aSyn increased the mean of the standard error of the mean (SEM) between ThT experiments compared to freezing (Figure 1B).

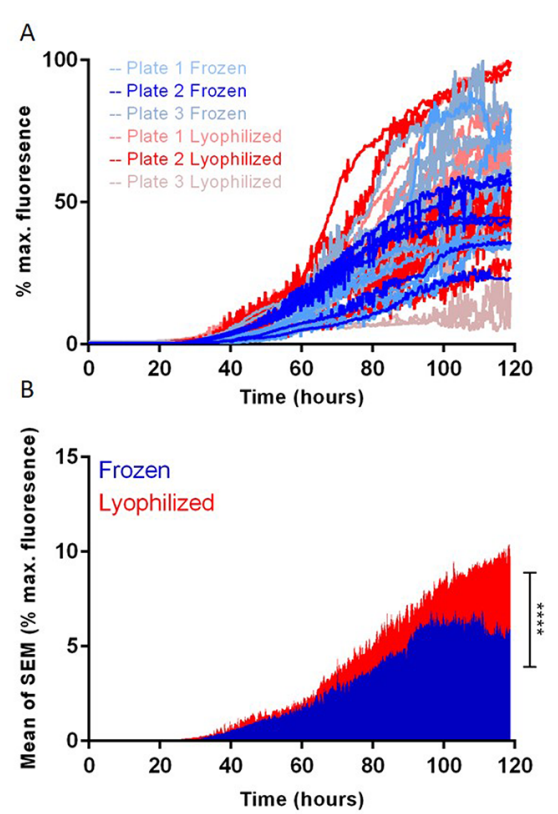

Figure 1. ThT-Based aggregation assay reveals variability in the aggregation kinetics of lyophilized and frozen aSyn. (A) Individual well ThT fluorescence intensity plotted as \% of maximum fluorescence per plate of lyophilized aSyn samples (red) and frozen aSyn samples (blue) over time. (B) Mean of the standard error of the mean (SEM) from three replicate plates with six wells containing frozen and lyophilized samples. Significant difference between the mean of SEMs of frozen and lyophilized samples is $p<0.0001$ using an unpaired $t$ test (Welch's). $100 \mu \mathrm{M}$ aSyn was incubated in a 96 well plate with continuous orbital agitation at $300 \mathrm{rpm}$ for $120 \mathrm{~h}$.
To determine the remaining monomer concentration of aSyn after the ThT-based aggregation assay we used analytical SEC and showed that there was less remaining monomeric aSyn in the lyophilized samples but a greater variability in well to well concentrations compared to frozen samples (Table S2). Rate constants for a 2-step (nucleation and elongation) aggregation mechanism were calculated using the Finke-Watzky equation ${ }^{30}$ from sigmoidal curves of the mean ThT fluorescence data (Figure S2). The nucleation rate, $\mathrm{A} \rightarrow \mathrm{B}$ (rate constant $k_{1}$ ) and the elongation rate $\mathrm{A}+\mathrm{B} \rightarrow 2 \mathrm{~B}$, (rate constant $k_{2}$ ) could be calculated from fitted ThT fluorescence curves (Figure S3), least-squares curve fitting was performed in MATLAB using curve fitting toolbox, goodness of fitted data is presented in Table S3. The $k_{1}$ nucleation rate was faster in the lyophilized aSyn sample, $1.22 \pm 0.1 \mathrm{~s}^{-1}$ nucleation events/s, compared to the frozen sample, $0.80 \pm 0.04 \mathrm{~s}^{-1}$ nucleation events/s (Table $2)$. Conversely, the $k_{2}$ elongation rate was slower for the lyophilized aSyn sample, $0.86 \pm 0.01 \mathrm{~ms}^{-1}$, compared to the frozen sample, $1.62 \pm 0.03 \mathrm{~ms}^{-1}$.

Table 2. Lyophilized and Frozen aSyn Display Different Aggregation Kinetics

$\begin{array}{ccc}\text { storage condition } & k_{1}\left(\mathrm{~s}^{-1}\right)^{a} & k_{2}\left(\mathrm{~ms}^{-1} \text { ThT fluorescence a.u. }\right)^{a} \\ \text { lyophilized } & 1.22 \pm 0.10 & 0.86 \pm 0.01 \\ \text { frozen } & 0.80 \pm 0.04 & 1.62 \pm 0.03\end{array}$

${ }^{a} k_{1}$ and $k_{2}$ values were calculated from fitting the Finke-Watzky equation to averaged ThT fluorescence curves. a.u. arbitrary units

Analytical Size Exclusion Chromatography of aSyn Identifies Mostly Monomeric Protein but High Molecular Weight Conformers Are Also Present in All Storage Conditions. To investigate the origin of the differences in variability and nucleation/elongation rates of lyophilized and frozen aSyn samples, we used a variety of structure sensitive methods, including analytical SEC, DLS, AFM, and HDX-MS, to deduce whether the choice of buffer and storage conditions had an effect on the protein conformation. Our analytical SEC results show that the majority of aSyn is in its monomeric form (Figure 2A), independent of the storage protocols used. Monomeric aSyn eluted at $6.78 \mathrm{~min}$ minutes from the calibrated BioSep-SEC-2000s column, from which a MW of $78.5 \pm 2.7 \mathrm{kDa}$ was estimated (Figure $2 \mathrm{~A}$ ). The predicted MW of aSyn using SEC has been previously shown to range between 60 and $70 \mathrm{kDa} .^{9,16,39}$ Monomeric aSyn in aqueous solution has an extended conformation, reflected in a larger hydrodynamic radius. The models used to calculate molecular weight from SEC data assume a globular fold and overestimate the mass of aSyn which is $14.4 \mathrm{kDa}$. The first elution peak, shown in the zoomed in Figure 2B, corresponds to high molecular weight (HMW) conformers of $480 \pm 17 \mathrm{kDa}$ (Figure 2B). Aggregates of similar size $(\sim 440 \mathrm{kDa}$; SDS-PAGE) have also been observed by others when aSyn was purified using precipitation by streptomycin sulfate and ammonium sulfate with subsequent lyophilization. ${ }^{40}$ aSyn samples that were lyophilized in $\mathrm{H}_{2} \mathrm{O}$ and reconstituted in $\mathrm{NaP}$ buffer had the highest content of HMW conformers, that is, $2.45 \pm 0.05 \%$. The aSyn samples lyophilized in $\mathrm{NaP}$ buffer had a lower percentage of HMW conformers of $0.12 \pm 0.01 \%$, but the lowest percentage of HMW conformers, $0.01 \pm 0.01 \%$, were found in the aSyn sample in $\mathrm{NaP}$ buffer which was directly frozen at $-80{ }^{\circ} \mathrm{C}$ (Table 3). 

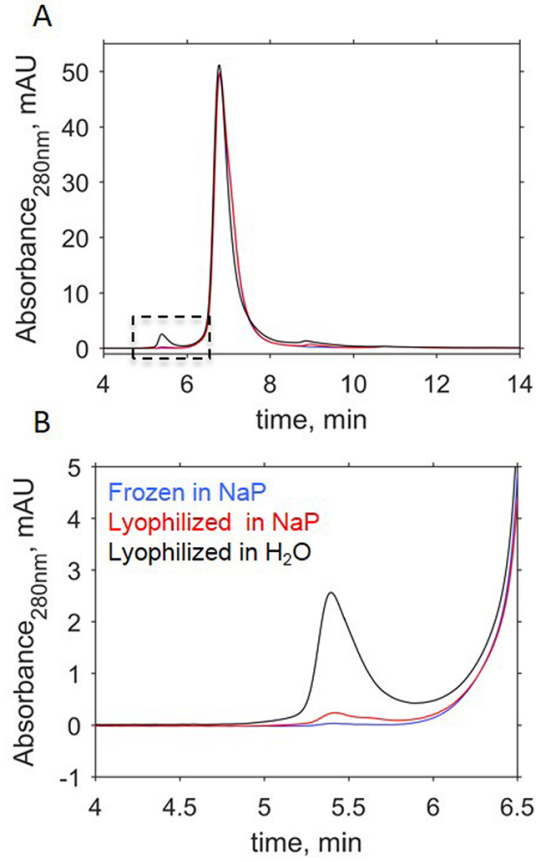

Figure 2. Analytical SEC reveals a higher degree of HMW conformers in the lyophilized aSyn samples. (A) Elution profile of aSyn after freezing in $\mathrm{NaP}$ (blue), lyophilizing in $\mathrm{NaP}$ (red), lyophilizing in $\mathrm{H}_{2} \mathrm{O}$ (black), all samples were filtered through a $0.22 \mu \mathrm{m}$ filter. $50 \mu \mathrm{L}$ of 100 $\mu \mathrm{M}$ aSyn was analyzed on a BioSep-SEC s2000 column equilibrated in PBS at $1 \mathrm{~mL} / \mathrm{min}$. Monomeric aSyn eluted from the column at $\sim 7$ min corresponding to MW $78.5 \pm 2.7 \mathrm{kDa}$. HMW conformers eluted earlier $\sim 5.5 \mathrm{~min}$, corresponding to MW $480 \pm 17 \mathrm{kDa}$. Dashed box outlines the zoomed in area shown in B. (B) Zoomed area shows more HMW conformers in the lyophilized samples compared to frozen, the most HMW conformers were detected in the lyophilized in $\mathrm{H}_{2} \mathrm{O}$ sample.

Table 3. Percentage of aSyn Monomer and HMW Conformers after Storage Determined by Analytical SEC

$\begin{array}{lcc}\text { storage protocol } & \text { monomer (\%) } & \text { HMW conformers (\%) } \\ \mathrm{H}_{2} \mathrm{O} \text { lyophilized } & 97.55 \pm 0.05 & 2.45 \pm 0.05 \\ \mathrm{NaP} \text { lyophilized } & 99.88 \pm 0.01 & 0.12 \pm 0.01 \\ \mathrm{NaP} \text { frozen } & 99.99 \pm 0.01 & 0.01 \pm 0.01\end{array}$

DLS Identifies a Wider Size Distribution of aSyn Monomeric Conformers after Lyophilizing than after Freezing. DLS was used to further characterize the size range of various conformers present in the $\mathrm{NaP}$ and $\mathrm{H}_{2} \mathrm{O}$ buffer samples. DLS can estimate the size distribution of particles in the nanometer-micrometer range based on their light scattering profiles. Previous reports show that monomeric aSyn has a diameter ranging between $4-8 \mathrm{~nm}$ using DLS. ${ }^{41}$ Small-angle X-ray scattering (SAXS) analysis also determined monomeric aSyn had a radius of $4.0 \pm 0.1 \mathrm{~nm}$, corresponding to a diameter of $\sim 8 \mathrm{~nm}$. A folded globular protein of the same length would have a diameter $\sim 3$, indicating that the monomeric aSyn is unfolded. ${ }^{33}$ Analyzing the DLS measured size distribution by volume, that is, analyzing primarily monomeric protein, we show that aSyn lyophilized in $\mathrm{NaP}$ buffer contained monomer which had a size distribution between $3-20 \mathrm{~nm}$, compared to $3-11 \mathrm{~nm}$ for aSyn frozen in $\mathrm{NaP}$ buffer (Figure 3). The size distributions may be attributed to by variations of monomer conformation and/or presence of some dimer/trimers. Analysis of the DLS data by intensity,

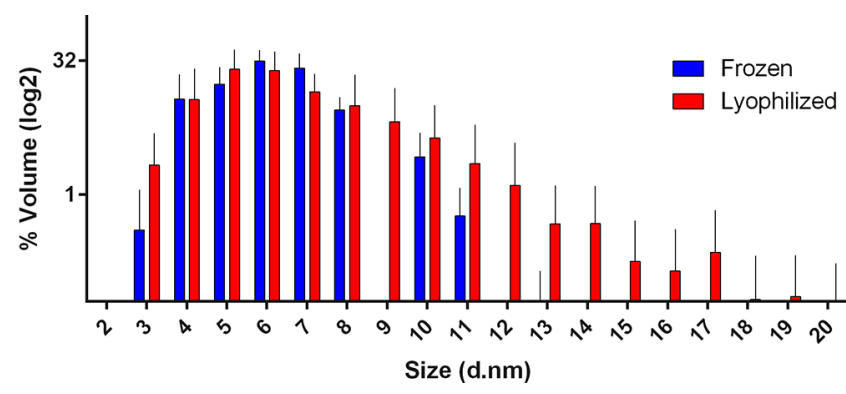

Figure 3. DLS reveals the presence of primarily monomeric protein in both frozen and lyophilized aSyn with the lyophilized sample displaying a wider size distribution. \% Volume $(\log 2)$ of the 100 $\mu \mathrm{M}$ aSyn sample is plotted against the diameter of aSyn detected (d.nm). aSyn frozen in NaP buffer (blue) and aSyn lyophilized in $\mathrm{H}_{2} \mathrm{O}$ and reconstituted in $\mathrm{NaP}$ buffer (red) contain primarily monomeric protein (4-8 d.nm). Next to monomeric protein there are also different aSyn conformers present, with the lyophilized sample displaying a greater size heterogeneity compared to the frozen sample. Experiments were repeated twice with a total of 35 readings for lyophilized samples and 21 for frozen samples.

focusing more on the HMW species, reveals HMW conformers of $200 \mathrm{~nm}$ in both lyophilized and frozen samples but also showing greater variance in the lyophilized aSyn by size distribution (Figure S4 and Table S4). Samples of aSyn lyophilized and frozen in $\mathrm{NaP}$ buffer were probed by AFM to examine the shape of the HMW conformers (Figure S5A and B). The conformers in both samples were heterogeneous in size and shape which has been also observed in other studies. ${ }^{42}$ However, the conformers induced by lyophilizing had a bigger surface area (Figure S5C) and showed greater heterogeneity in shape, while frozen aSyn HMW conformers were more ellipsoid in shape (Figure S5A and B).

HDX-MS Confirms the Presence of Different Monomeric Conformers in the Lyophilized versus Frozen aSyn Samples. The above analytical SEC and DLS results have identified that the majority of the aSyn protein was in its monomeric form, but there may be different monomeric conformations present after freezing and lyophilization. To probe the submolecular conformation of aSyn in its monomeric form and to measure the impact of freezing and lyophilization on the monomer, we applied HDX-MS. The observed rates of $\mathrm{H} / \mathrm{D}$-exchange indicate the degree of solvent exposure and/or stable hydrogen-bonding. Prior to HDX-MS, the sample lyophilized in $\mathrm{NaP}$ buffer was freshly dissolved into $\mathrm{H}_{2} \mathrm{O}$ and both the frozen and lyophilized samples were diluted to a stock concentration of $80 \mu \mathrm{M}$. HDX-MS was done as a "bottom-up" experiment: therefore, the protein sequence was initially mapped to the experimental data (essentially a spectral assignment process) in order to confidently monitor H/Dexchange at the submolecular level. The assigned peptides from the mass spectra are the basis for submolecular resolution, which is summarized in a coverage map (Figure S6). The protein was then labeled with deuterium by incubation at $\mathrm{pH}$ 4.00 for various time intervals, ranging from $30 \mathrm{~s}$ to $50 \mathrm{~min}$ (3000 s). The labeling experiment was performed at $\mathrm{pH} 4.00$, as the kinetics of $\mathrm{H} / \mathrm{D}$ exchange were too fast to observe any difference at $\mathrm{pH} 7.2$ (Figure S7). This is a reflection of the very low hydrogen-exchange protection factors in an intrinsically disordered protein, such as aSyn. The HDX-MS data are obtained from a time course study of deuterium uptake at each measured location within the protein chain. The data are 


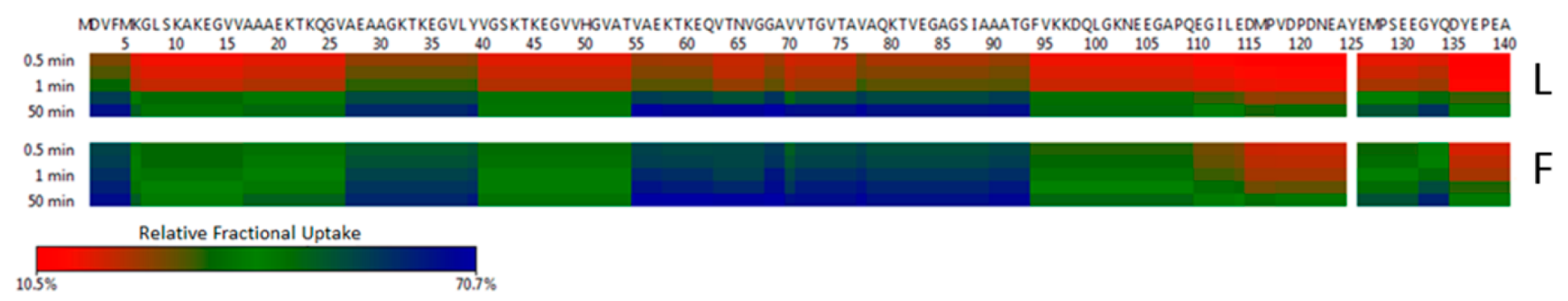

Figure 4. Relative fractional deuterium uptake for frozen and lyophilized aSyn samples. Lyophilized aSyn is more solvent protected than frozen aSyn but both samples contain a solvent protected region at the C-terminus. Shown is the relative fractional mass increase over the time course of the experiment, samples were taken at $0.5,0.75,1,5$, and $50 \mathrm{~min}$. Data is scaled between the minimum and maximum values observed. Large values of relative uptake (blue) indicate solvent accessibility or lack of stable hydrogen bonding, while small values (red) indicate solvent protection and/or stable hydrogen bonding.

expressed as a percentage of the maximum possible deuterium uptake at each site-this is termed the relative fractional uptake. Analysis of the relative fractional uptake of deuterium in both the lyophilized and frozen samples showed that the NAC region had the most deuterium uptake both in terms of exchange rate and the observed extent of labeling after $50 \mathrm{~min}$ (Figure 4). Two regions were observed in the $\mathrm{N}$-terminal domain that showed a high degree of protection to $\mathrm{H} / \mathrm{D}$ exchange (residues 5-26 and 40-55); both with even more pronounced protection following lyophilization. The greatest degree of protection against deuterium-labeling was observed in two regions in the C-terminal domain (residues 115-125 and 135-140). The protection from deuterium uptake observed at the $\mathrm{C}$-terminus under both conditions suggests there is a $\mathrm{C}$ terminal fold in the monomeric protein. This has not been reported previously using HDX-MS, although other biophysical studies have observed an overall compaction of aSyn at lower $\mathrm{pH}^{33,43,44}$ When comparing relative differences in deuterium uptake between lyophilized and frozen samples, the lyophilized sample was found to exchange at a slower rate than the frozen sample throughout the protein sequence. This is most evident in the reduced relative fractional uptake at early time points (Figure 4) and results in a positive value for the difference of mean deuteration (Figure S8). This indicates increased solvent protection, or the emergence of secondary structure. We interpret this as a compacting of the lyophilized sample through increased hydrogen bonding either internally or externally to other monomers. Importantly, although it has previously been shown that lyophilization may induce aggregation (shown here by DLS and AFM), HDX-MS data indicate that lyophilization also affects the structure of monomeric protein.

\section{DISCUSSION}

There has been an extensive research effort in the field of amyloid protein aggregation to determine the kinetics of fibril formation and to understand how toxic forms of amyloid proteins in disease arise. Although we are slowly unpicking the biophysical mechanisms behind monomer to fibril formation, there are still a great number of variables, such as the effect of buffer solution and protein storage protocols that need to be taken into consideration and which may hamper our goal to reliably measure amyloid protein aggregation kinetics. In this study, we deduce that for aSyn, freezing is preferential to lyophilization which not only introduces more HMW conformers but also has an effect on the monomeric protein as shown by HDX-MS. Note, we use the term "conformers" to describe aSyn species because (a) we cannot conclude that the aSyn species we observe are similar to "oligomers" referred to in the literature, particularly HMW conformers formed during lyophilization as these seem to have different morphology as determined by AFM to those formed by incubation of high concentrations of $\mathrm{aSyn}^{45}$ and (b) we already observe a structural conformer in monomeric aSyn which may directly influence aggregation kinetics. Because of the very low concentrations of HMW conformers in our samples, we were unable to isolate them for further structural analysis or to identify whether they became incorporated into fibrils or if they even could inhibit elongation.

Many protocols rely on lyophilization as a method for protein storage. We show, as part of the study, that lyophilizing aSyn greatly affects the variability in a ThT-based aggregation assay. In particular, lyophilization causes an increase in the percentage of HMW conformers, increases the heterogeneity of conformers, and most importantly affects the monomer structure by altering the hydrogen bonding, possibly by compaction or by inducing intermolecular bonding. As discussed, we observed differences in nucleation and elongation rates for frozen and lyophilized aSyn samples, however, it is difficult to speculate why the mechanism of fibrillization may be different since we do not have single fibril resolution. Using a single fibril-based aggregation assay, we and others have previously shown that there is structural polymorphism in aSyn fibrils formed in vitro as certain aSyn fibrils had failed to elongate in a seeding assay, while others had monodirectional and bidirectional growth. ${ }^{46-48}$ Whether or not this polymorphism stems from the presence of different monomeric aSyn conformers present at the start of the experiment or from the presence of certain HMW conformers remains to be determined.

Despite lyophilization increasing the number of HMW conformers in the samples compared to freezing, the total amount of HMW species formed in all samples was relatively low, ranging from $2.45 \%$ in $\mathrm{H}_{2} \mathrm{O}$ to $0.12-0.01 \%$ in $\mathrm{NaP}$ buffer. However, lyophilizing the protein in $\mathrm{H}_{2} \mathrm{O}$ rather than in $\mathrm{NaP}$ buffer does increase the percentage of HMW conformers present. DLS revealed that there is an increase in the heterogeneity of the size distribution of conformers formed during lyophilization compared to freezing. In particular, using AFM, we could show that the HMW conformers formed during lyophilizing were heterogeneous in shape, while those formed during freezing displayed a more homogeneous ellipsoid shape. This ellipsoid shape has been reported by others using oligomers formed during incubation of highly concentrated aSyn and may suggest that lyophilization is introducing nonphysiological HMW conformers. $95,45,49,50$ 
To gain more insight into the structural differences of the primarily monomeric protein, we applied HDX-MS which provides molecular-scale resolution. We observed the highest solvent protection at the C-terminus of aSyn in both, the lyophilized and the frozen aSyn sample. We also observed that the NAC region was the least protected region of the protein. Previously published HDX-MS data on aSyn probed oligomeric aSyn, which had already been aggregated, showing NAC protection and C-terminus exposure. ${ }^{51,52} \mathrm{We}$, however, expect our results to stem from monomeric protein due to the very low percentage of HMW conformers present in our samples, $0.12 \%$ in lyophilized and $0.01 \%$ in frozen samples.

So far HDX-MS had not been used to study the structure of monomeric aSyn. There have though been studies using NMR suggesting that there are certain intramolecular interactions present in the monomeric form of aSyn. Nuclear magnetic resonance with paramagnetic relaxation enhancement (NMR PRE) studies have proposed that the NAC region of aSyn may be protected from intermolecular interactions and aggregation in the monomeric form of aSyn, as the charged C-terminus can fold over and interact with the hydrophobic NAC region. ${ }^{53-55}$ However, there are other studies which have identified $\mathrm{N}$ terminus residues $12-26^{53}$ and residues $3-15$ and $35-50^{56}$ directly interacting with the $\mathrm{C}$ - terminus of aSyn. Again, others propose it is both a combination of hydrophobic C-terminus and $\mathrm{NAC}$, and $\mathrm{N}$ - and $\mathrm{C}$-terminus charge interactions that determine the structure of the monomer. ${ }^{57,58}$ Our HDX-MS data suggest that the $\mathrm{C}$-terminus may interact with the $\mathrm{N}$ terminus since we see a higher degree of HDX-MS protection at residues 5-26 and 40-55, rather than within the NAC region where we observe less protection. The most striking observation has been that the highest level of protection occurs at the C-terminus. Bertoncini et al., had previously identified two hydrophobic regions of aSyn at residues 115-119 and 125-129 within the C-terminus using NMR. ${ }^{53}$ This combined with our HDX-MS results, where we observe significant hydrogen-exchange protection for residues 115-125 and 135-140, suggests that there is a fold at the C-terminus.

We propose HDX-MS as a useful method to analyze monomeric IDPs, particularly as the field advances into fast mixing allowing millisecond sampling to capture folding events and study misfolding pathways. Currently, many studies utilize NMR PRE to study intramolecular interactions of IDPs. This method requires labeled isotopes and a high concentration of protein. HDX-MS on the other hand only uses low protein concentrations and, in comparison, is higher through-put.

Whether the aSyn conformations identified in the present study are involved in elongation or toxicity is a very pertinent question in the field, particularly in light of the growing evidence of the presence of different subpopulations of oligomers and polymorphs of aSyn fibrils, the formation of which being strongly dependent on their surrounding environment, ${ }^{59-61}$ but also in light of the debate within the field of residual structure in the "disordered" monomer. ${ }^{53,60,62}$ We show in this work that it is important to fully characterize the amyloid protein of interest within the experimental setup, i.e. considering buffer and storage conditions before studying amyloid aggregation kinetics. Only if we know what the starting conditions are will we be able to improve sample to sample variability in the future and further unpick the biophysical mechanisms of folding, ultimately advancing the future of therapeutic strategies.

\section{ASSOCIATED CONTENT}

\section{S Supporting Information}

The Supporting Information is available free of charge on the ACS Publications website at DOI: 10.1021/acs.analchem.8b01264.

Analysis of aSyn purity by Coomassie stained SDS-PAGE gel and analytical RP-HPLC, different aggregation kinetics measured for frozen and lyophilized aSyn, fitted curves calculated using the Finke Watzky equation to average ThT fluorescence data from lyophilized and frozen aSyn, DLS experiments displayed as percentage intensity versus size show a higher percentage of HMW conformers after lyophilizing than after and freezing, AFM reveals different aSyn HMW conformers after lyophilizing and freezing, comparison of the relative deuterium uptake (Da) for aSyn (resides 77-89) lyophilized and frozen samples over exposure time at $\mathrm{pH} 4$ and 7 reveals differences between monomeric lyophilized and frozen aSyn, coverage map of $\alpha$ synucleinpeptides from HDX-MS, difference in deuterium uptake between the aSyn frozen and aSyn lyophilized samples, amino acid verification of purified human aSyn, remaining aSyn monomer concentration taken at the end of the ThT-based aggregation assay and determined by analytical SEC is reduced in lyophilized samples but displays an increased variance between wells, goodness of fit represented by $R^{2}$ and root mean square error calculated from fitted curves of lyophilized and frozen aSyn samples, and DLS analysis of size distribution and percent intensity of aSyn reveals a higher degree of HMW conformers in lyophilized versus frozen samples (PDF)

\section{AUTHOR INFORMATION}

\section{Corresponding Author}

*E-mail: gsk20@cam.ac.uk.

ORCID

Amberley D. Stephens: 0000-0002-7303-6392

Jonathan J. Phillips: 0000-0002-5361-9582

Gabriele S. Kaminski Schierle: 0000-0002-1843-2202

\section{Author Contributions}

A.D.S. and N.N. contributed equally. A.D.S., N.N., M.Z., and J.J.P performed experiments and analyzed data. A.D.S, N.N., M.Z., J.J.P., C.F.K., and G.S.K.S. contributed to manuscript writing. All authors have given approval to the final version of the manuscript.

Notes

The authors declare no competing financial interest.

\section{ACKNOWLEDGMENTS}

G.S.K.S. and C.F.K. acknowledge funding from the Wellcome Trust, the UK Medical Research Council (MRC), Alzheimer Research UK (ARUK), and Infinitus China Ltd. C.F.K. acknowledges funding from the UK Engineering and Physical Sciences Research Council (EPSRC). M.Z. acknowledges funding from the Eugenides Foundation, Newnham College (Cambridge), and George and Marie Vergottis Foundation (Cambridge Trust). A.D.S. and M.Z. acknowledge the European Biophysical Societies' Association (EBSA), Alzheimer Research UK (ARUK), Newnham College (Cambridge) and British Mass Spectrometry Society (BMSS) for travel grants. 


\section{REFERENCES}

(1) Ramirez-Alvarado, M. Curr. Top. Med. Chem. 2013, 12, 2523-33.

(2) Sweeney, P.; Park, H.; Baumann, M.; Dunlop, J.; Frydman, J.; Kopito, R.; McCampbell, A.; Leblanc, G.; Venkateswaran, A.; Nurmi, A.; Hodgson, R. Transl. Neurodegener. 2017, 6, 6.

(3) Mukherjee, A.; Morales-Scheihing, D.; Butler, P. C.; Soto, C. Trends Mol. Med. 2015, 21, 439-449.

(4) Knowles, T. P. J.; Mezzenga, R. Adv. Mater. 2016, 28, 65466561 .

(5) Bolognesi, B.; Kumita, J. R.; Barros, T. P.; Esbjorner, E. K.; Luheshi, L. M.; Crowther, D. C.; Wilson, M. R.; Dobson, C. M.; Favrin, G.; Yerbury, J. J. ACS Chem. Biol. 2010, 5, 735-740.

(6) Pinotsi, D.; Buell, A. K.; Dobson, C. M.; Kaminski Schierle, G. S.; Kaminski, C. F. ChemBioChem 2013, 14, 846-850.

(7) Chan, F. T. S.; Kaminski Schierle, G. S.; Kumita, J. R.; Bertoncini, C. W.; Dobson, C. M.; Kaminski, C. F. Analyst 2013, 138, 2156.

(8) Giehm, L.; Otzen, D. E. Anal. Biochem. 2010, 400, 270-281.

(9) Lorenzen, N.; Nielsen, S. B.; Buell, A. K.; Kaspersen, J. D.; Arosio, P.; Vad, B. S.; Paslawski, W.; Christiansen, G.; ValnickovaHansen, Z.; Andreasen, M.; Enghild, J. J.; Pedersen, J. S.; Dobson, C. M.; Knowles, T. P. J.; Otzen, D. E. J. Am. Chem. Soc. 2014, 136, 38593868 .

(10) Paslawski, W.; Lorenzen, N.; Otzen, D. E. Methods Mol. Biol. 2016, 1345, 133-150.

(11) Stefanis, L. Cold Spring Harbor Perspect. Med. 2012, 2, a009399.

(12) Huang, C.; Ren, G.; Zhou, H.; Wang, C. Protein Expression Purif. 2005, 42, 173-177.

(13) Campioni, S.; Carret, G.; Jordens, S.; Nicoud, L.; Mezzenga, R.; Riek, R. J. Am. Chem. Soc. 2014, 136, 2866-2875.

(14) Giehm, L.; Lorenzen, N.; Otzen, D. E. Methods 2011, 53, 295305.

(15) Jakes, R.; Spillantini, M. G.; Goedert, M. FEBS Lett. 1994, 345, $27-32$.

(16) Weinreb, P. H.; Zhen, W.; Poon, A. W.; Conway, K. A.; Lansbury, P. T. Biochemistry 1996, 35, 13709-13715.

(17) Conway, K. A.; Harper, J. D.; Lansbury, P. T. Nat. Med. 1998, 4, 1318

(18) Narhi, L.; Wood, S. J.; Steavenson, S.; Jiang, Y.; Wu, G. M.; Anafi, D.; Kaufman, S. A.; Martin, F.; Sitney, K.; Denis, P.; Louis, J.-C.; Wypych, J.; Biere, A. L.; Citron, M. J. Biol. Chem. 1999, 274, 98439846.

(19) Grey, M.; Linse, S.; Nilsson, H.; Brundin, P.; Sparr, E. J. Parkinsons. Dis. 2011, 1, 359-371.

(20) Carpenter, J. F.; Chang, B. S.; Garzon-Rodriguez, W.; Randolph, T. W. Pharm. Biotechnol. 2002, 13, 109-133.

(21) Nail, S. L.; Jiang, S.; Chongprasert, S.; Knopp, S. A. In Development and Manufacture of Protein Pharmaceuticals; Nail, S. L., Akers, M. J., Eds.; Springer US: Boston, MA, 2002; pp 281-360.

(22) Pikal-Cleland, K. A.; Rodríguez-Hornedo, N.; Amidon, G. L.; Carpenter, J. F. Arch. Biochem. Biophys. 2000, 384, 398-406.

(23) van den Berg, L.; Rose, D. Arch. Biochem. Biophys. 1959, 81, 319-329.

(24) Costantino, P. D.; Henry, R, Michael, J.; Pikal Lyophilization of Biopharmaceuticals; AAPS Press, 2004.

(25) Norde, W. Cells Mater. 1995, 5, 97-112.

(26) Heller, M. C.; Carpenter, J. F.; Randolph, T. W. Biotechnol. Bioeng. 1999, 63, 166-174.

(27) Bhatnagar, B. S.; Bogner, R. H.; Pikal, M. J. Pharm. Dev. Technol. 2007, 12, 505-523.

(28) Prestrelski, S. J.; Tedeschi, N.; Arakawa, T.; Carpenter, J. F. Biophys. J. 1993, 65, 661-671.

(29) Schindelin, J.; Arganda-Carreras, I.; Frise, E.; Kaynig, V.; Longair, M.; Pietzsch, T.; Preibisch, S.; Rueden, C.; Saalfeld, S.; Schmid, B.; Tinevez, J.-Y.; White, D. J.; Hartenstein, V.; Eliceiri, K.; Tomancak, P.; Cardona, A. Nat. Methods 2012, 9, 676-682.

(30) Watzky, M. A.; Finke, R. G. J. Am. Chem. Soc. 1997, 119, $10382-10400$

(31) Munishkina, L. A.; Henriques, J.; Uversky, V. N.; Fink, A. L. Biochemistry 2004, 43, 3289-3300.
(32) Binolfi, A.; Rasia, R. M.; Bertoncini, C. W.; Ceolin, M.; Zweckstetter, M.; Griesinger, C.; Jovin, T. M.; Fernández, C. O. J. Am. Chem. Soc. 2006, 128, 9893-9901.

(33) Uversky, V. N.; Li, J.; Fink, A. L. J. Biol. Chem. 2001, 276, 10737-10744.

(34) Uversky, V. N.; Li, J.; Souillac, P.; Millett, I. S.; Doniach, S.; Jakes, R.; Goedert, M.; Fink, A. L. J. Biol. Chem. 2002, 277, 1197011978.

(35) Buell, A. K.; Galvagnion, C.; Gaspar, R.; Sparr, E.; Vendruscolo, M.; Knowles, T. P. J.; Linse, S.; Dobson, C. M. Proc. Natl. Acad. Sci. U. S. A. 2014, 111, 7671-7676.

(36) Nilsson, M. R. Methods 2004, 34, 151-160.

(37) Groenning, M. J. Chem. Biol. 2010, 3, 1-18.

(38) Biancalana, M.; Koide, S. Biochim. Biophys. Acta, Proteins Proteomics 2010, 1804, 1405-1412.

(39) Coelho-Cerqueira, E.; Carmo-Gonçalves, P.; Sá Pinheiro, A.; Cortines, J.; Follmer, C. FEBS J. 2013, 280, 4915-4927.

(40) Celej, M. S.; Sarroukh, R.; Goormaghtigh, E.; Fidelio, G. D.; Ruysschaert, J.-M.; Raussens, V. Biochem. J. 2012, 443, 719-26.

(41) Horvath, I.; Sellstedt, M.; Weise, C.; Nordvall, L.-M.; Krishna Prasad, G.; Olofsson, A.; Larsson, G.; Almqvist, F.; Wittung-Stafshede, P. Arch. Biochem. Biophys. 2013, 532, 84-90.

(42) Ghosh, D.; Singh, P. K.; Sahay, S.; Jha, N. N.; Jacob, R. S.; Sen, S.; Kumar, A.; Riek, R.; Maji, S. K. Sci. Rep. 2015, 5, 9228.

(43) Phillips, A. S.; Gomes, A. F.; Kalapothakis, J. M. D.; Gillam, J. E.; Gasparavicius, J.; Gozzo, F. C.; Kunath, T.; MacPhee, C.; Barran, P. E. Analyst 2015, 140, 3070-3081.

(44) Bernstein, S. L.; Liu, D.; Wyttenbach, T.; Bowers, M. T.; Lee, J. C.; Gray, H. B.; Winkler, J. R. J. Am. Soc. Mass Spectrom. 2004, 15, $1435-1443$.

(45) Apetri, M. M.; Maiti, N. C.; Zagorski, M. G.; Carey, P. R. Anderson, V. E. J. Mol. Biol. 2006, 355, 63-71.

(46) Pinotsi, D.; Michel, C. H.; Buell, A. K.; Laine, R. F.; Mahou, P.; Dobson, C. M.; Kaminski, C. F.; Kaminski Schierle, G. S. Proc. Natl. Acad. Sci. U. S. A. 2016, 113, 3815.

(47) Wördehoff, M. M.; Bannach, O.; Shaykhalishahi, H.; Kulawik, A.; Schiefer, S.; Willbold, D.; Hoyer, W.; Birkmann, E. J. Mol. Biol. 2015, 427, 1428-1435.

(48) Pinotsi, D.; Buell, A. K.; Galvagnion, C.; Dobson, C. M.; Kaminski Schierle, G. S.; Kaminski, C. F. Nano Lett. 2014, 14, 339345.

(49) Chen, S. W.; Drakulic, S.; Deas, E.; Ouberai, M.; Aprile, F. A.; Arranz, R.; Ness, S.; Roodveldt, C.; Guilliams, T.; De-Genst, E. J.; Klenerman, D.; Wood, N. W.; Knowles, T. P. J.; Alfonso, C.; Rivas, G.; Abramov, A. Y.; Valpuesta, J. M.; Dobson, C. M.; Cremades, N. Proc. Natl. Acad. Sci. U. S. A. 2015, 112, E1994-2003.

(50) Conway, K. A.; Lee, S.-J.; Rochet, J.-C.; Ding, T. T.; Williamson, R. E.; Lansbury, P. T. Proc. Natl. Acad. Sci. U. S. A. 2000, 97, 571-576.

(51) Mysling, S.; Betzer, C.; Jensen, P. H.; Jorgensen, T. J. D. Biochemistry 2013, 52, 9097-9103.

(52) Paslawski, W.; Mysling, S.; Thomsen, K.; Jørgensen, T. J. D.; Otzen, D. E. Angew. Chem., Int. Ed. 2014, 53, 7560-7563.

(53) Bertoncini, C. W.; Jung, Y.-S.; Fernandez, C. O.; Hoyer, W.; Griesinger, C.; Jovin, T. M.; Zweckstetter, M. Proc. Natl. Acad. Sci. U. S. A. 2005, 102, 1430-1435.

(54) Fernández, C. O.; Hoyer, W.; Zweckstetter, M.; Jares-Erijman, E. A.; Subramaniam, V.; Griesinger, C.; Jovin, T. M. EMBO J. 2004, 23, 2039-2046

(55) Dedmon, M. M.; Lindorff-Larsen, K.; Christodoulou, J.; Vendruscolo, M.; Dobson, C. M. J. Am. Chem. Soc. 2005, 127, 476477.

(56) Wu, K. P.; Baum, J. J. Am. Chem. Soc. 2010, 132, 5546-5547.

(57) Zhou, W.; Long, C.; Reaney, S. H.; Di Monte, D. A.; Fink, A. L.; Uversky, V. N. Biochim. Biophys. Acta, Mol. Basis Dis. 2010, 1802, 322330.

(58) Hong, D.-P.; Xiong, W.; Chang, J.-Y.; Jiang, C. FEBS Lett. 2011, $585,561-566$

(59) Roeters, S. J.; Iyer, A.; Pletikapić, G.; Kogan, V.; Subramaniam, V.; Woutersen, S. Sci. Rep. 2017, 7, 41051. 
(60) Bousset, L.; Pieri, L.; Ruiz-Arlandis, G.; Gath, J.; Jensen, P. H.; Habenstein, B.; Madiona, K.; Olieric, V.; Böckmann, A.; Meier, B. H.; Melki, R. Nat. Commun. 2013, 4, 2575.

(61) Ranjan, P.; Kumar, A. ACS Chem. Neurosci. 2017, 8, 2235.

(62) Nugent, E.; Kaminski, C. F.; Kaminski Schierle, G. S. Integr. Biol. 2017, 9, 206-210. 